\title{
Interfaces entre lombalgia e envelhecimento
}

\section{Interface between back pain and aging}

\author{
Jonathan Loro Pessin', Ângelo José Gonçalves Bos²
}

${ }^{1}$ Quiropraxista especialista. Instituto de Ciências da Saúde, Universidade Feevale, Novo Hamburgo, RS, Brasil. <jloropessin@yahoo.com.br>

2 Médico PhD. Instituto de Geriatria e Gerontologia, Pontifícia Universidade Católica do Rio Grande do Sul, Porto Alegre, RS, Brasil. <angelo.bos@pucrs.br>

\section{ARTICLE INFO}

\section{Article history}

Received: 19/08/2016

Accepted: 07/11/2016

\section{Correspondent Author}

Jonathan Loro Pessin

Rua Júlio de Castilhos, 1 123/603

Farroupilha, RS, Brasil

<jloropessin@yahoo.com.br>

\section{(C) 2016 All rights reserved}

\section{Editors}

Alfredo Cataldo Neto

Paula Engroff

\begin{abstract}
RESUMO
Objetivos: Foi realizada uma revisão narrativa e compreensiva de estudos e pesquisas sobre o fenômeno da dor lombar e seu acometimento no processo do envelhecimento humano. Fonte de dados: Foram pesquisadas as seguintes bases de dados: MEDLINE, SciELO, Bireme e LILACS, através dos termos "lombalgia", "lombalgia senil", "dor lombar e idoso"; "lombalgia e idoso"; "dor lombar e envelhecimento", referentes ao período de 1996 a 2012. Síntese dos dados: Foram selecionadas e analisadas 27 referências, que mostraram forte relação entre a lombalgia e declínio na qualidade de vida do indivíduo idoso, que resulta da interação de fatores sociodemográficos (idade, sexo, renda e escolaridade), comportamentais (tabagismo e sedentarismo), atividades cotidianas (trabalho físico pesado, vibração, posição viciosa e movimentos repetitivos) e outros (obesidade e morbidade psicológica). Conclusões: Apesar da magnitude do impacto social e econômico, apontada em estudos internacionais, no Brasil há escassez de estudos sobre prevalência de lombalgia em idosos. Esse fato dificulta a sensibilização de profissionais da área de saúde, a alocação de recursos humanos e materiais, e a criação de estratégias visando ao controle da dor lombar nessa população, tornando emergente a necessidade de estudos epidemiológicos com este enfoque.
\end{abstract}

PALAVRAS-CHAVE: Idoso; Dor Lombar; Envelhecimento; Revisão.

\begin{abstract}
Aims: It was realized a narrative and comprehensive review with studies and researches about the phenomenon of low back pain and its involvement in the process of human aging. Source of data: It were used the following databases: MEDLINE, SciELO, LILACS and Bireme, with the mesh terms "low back pain", "senile low back pain" e "chronic low back pain", for the period from 1996 to 2012. Summary of findings: Were selected and analyzed 27 references, which showed a strong relation between low back pain and decreased quality of life of the elderly, which results from the interaction of sociodemographic factors (age, sex, income and education), behavioral (smoking and physical inactivity), daily activities (heavy physical work, vibration, vicious posture and repetitive movements) and other factors (obesity and psychological morbidity). Conclusions: Despite the magnitude of the social and economic impact, pointed in international studies, in Brazil there are few studies on the prevalence of low back pain in older adults. This fact complicates the awareness of health professionals, the allocation of human and material resources, and the creation of strategies for the control of low back pain in this population, making the emerging need for epidemiological studies with this approach.

KeYwords: Aged; Low Back Pain; Aging; Review.
\end{abstract}




\section{INTRODUÇÃO}

O envelhecimento pode ser considerado como um conjunto de alterações estruturais e funcionais do organismo humano, decorrente de acúmulos fisiológicos que ocorrem de forma progressiva. Caracteriza um processo de mudanças contínuas, que vai desde o declínio fisiológico gradual ao aumento de doenças que acometem o organismo paralelamente. ${ }^{1,2}$ Com o avanço da idade, os indivíduos tornam-se mais sedentários, resultando numa redução de suas capacidades físicas. A diminuição das atividades físicas resulta na aparição de agravos que contribuem para deterioração do processo de envelhecimento. ${ }^{3,4}$

Apesar de o envelhecimento não estar, necessariamente, relacionado às doenças e incapacidades, o idoso, por ser vítima de mecanismos fisiológicos que alteram a sua capacidade física, torna-se um forte candidato a apresentar queixas de lombalgia. Um estudo de revisão sistemática e metanálise aponta que um em cada quatro idosos apresenta lombalgia em um dado momento do tempo. ${ }^{5}$ A lombalgia é um sintoma referido na altura da cintura pélvica, gerando um quadro clínico de dor, incapacidade de se movimentar e trabalhar, representando, desta forma, uma grande causa de morbidade e incapacidade dentro dos distúrbios dolorosos que afetam o homem. ${ }^{6}$

Predominantemente no idoso tem início insidioso e é causada pela degeneração de estruturas da coluna vertebral inerente ao processo de envelhecimento, que gera alterações nas partes ósseas (achatamento dos corpos vertebrais e perda de massa óssea, que poderá predispor a fraturas), além de modificações discais e ligamentares da coluna vertebral. Embora um episódio isolado possa ter recuperação espontânea ou diminuir sua intensidade, este tipo de dor é em geral uma condição recorrente que frequentemente evolui para um estado crônico. Do ponto de vista evolutivo, a dor lombar é considerada aguda quando tem duração menor que seis semanas, subaguda entre 6 a 12 semanas, e crônica com mais do que 12 semanas. ${ }^{6}$

As dores lombares podem ser primárias ou secundárias, com ou sem envolvimento neurológico (lombociatalgias) e podem ser causadas por patologias inflamatórias, degenerativas, neoplásicas, defeitos congênitos, déficit muscular, predisposição reumática e outras. ${ }^{7,8}$ No entanto, esse quadro patológico pode estar associado também a múltiplas causas como, por exemplo, fatores sociodemográficos (idade, sexo, renda e escolaridade), comportamentais (tabagismo e sedentarismo), fatores encontrados nas atividades cotidianas (trabalho físico pesado, vibração, posição viciosa e movimentos repetitivos) e outros (obesidade e morbidade psicológica). ${ }^{6}$

O bem-estar físico e a qualidade de vida do idoso são comprometidos quando a dor lombar se torna persistente, podendo gerar ou agravar a depressão. Restrições para a realização das atividades de vida diária, diminuição da mobilidade, problemas no sono e no apetite são algumas consequências ocasionadas por esta queixa referida entre idosos. ${ }^{4}$

Atualmente 33\% dos idosos apresentam lombalgia, seja por causa desconhecida, por dor crônica ou irradiação para os membros inferiores. ${ }^{9}$ Apesar dos diversos estudos que quantificam a prevalência da lombalgia em grupos populacionais determinados, há escassez de material publicado especificamente na população idosa. ${ }^{6}$ Dentro deste contexto o objetivo do presente estudo foi analisar as relações entre a lombalgia e o processo de envelhecimento, bem como traçar os prejuízos resultantes desta associação a partir da literatura indexada em bases de dados nacionais e internacionais.

\section{REVISÃO DA LITERATURA}

Foi realizada uma revisão narrativa e compreensiva de estudos e pesquisas sobre o fenômeno da dor lombar e seu acometimento no processo do envelhecimento humano. Considerou-se a tendência recente de buscar a força das evidências em diferentes fontes, abordagens e metodologias em estudos realizados especialmente em países em desenvolvimento.

Estudos clínicos e randomizados, pautados na força da validade interna ou na efetividade e evidenciados pelos dados quantitativos, foram bastante explorados. Foram selecionados estudos que tratassem da lombalgia em termos epidemiológicos, bem como sua relação com o indivíduo idoso e consequências no processo de envelhecimento em geral.

As referências apresentadas pela literatura foram coletadas a partir das bases de dados MEDLINE, SciELO, Bireme e LILACS. As palavras-chave e termos utilizados na busca revisão foram, em inglês: "low back pain", "senile low back pain" e "chronic low back pain". Pesquisaram-se também os termos, em português: "lombalgia", "lombalgia senil", "dor lombar e idoso"; "lombalgia e idoso"; "dor lombar e envelhecimento".

O estudo abrangeu publicações partir dos anos 1996 até 2012. A base de maior relevância para o trabalho foi Scielo, seguida da Bireme. Foram analisadas 39 referências, sendo 27 sobre o tema proposto; as 12 restantes referiam metodologia a respeito de relação entre dor lombar e idade infantil ou adulta, lombalgia e tratamento cirúrgico ou lombalgia e sua relação com 
as mais diversas patologias, não atendendo diretamente ao objetivo deste estudo, sendo assim descartadas.

Os dados foram sistematizados em três categorias: A dor no indivíduo idoso; Dor lombar e envelhecimento; e Fatores sociais e incapacidades decorrentes da lombalgia.

\section{A dor no indivíduo idoso}

A transição demográfica é um fenômeno de amplitude mundial, assim, a longevidade é um fenômeno que está também presente na sociedade brasileira. ${ }^{10} \mathrm{O}$ processo de envelhecimento, na maioria das vezes, não se caracteriza como um período saudável e de independência. Ao contrário, caracteriza-se pela alta incidência de doenças crônicas e degenerativas que, muitas vezes, resultam em elevada dependência. Muitos desses quadros são acompanhados por dor e, em significativa parcela deles, a dor crônica é a principal queixa do indivíduo, fato que pode interferir de modo acentuado na qualidade de vida dos idosos. ${ }^{11}$

A dor é considerada pela Associação Internacional de Estudos da Dor (IASP) como uma experiência desagradável, sensitiva e emocional, associada ou não ao dano real ou potencial de lesões dos tecidos e relacionada com a memória individual, com as expectativas e as emoções de cada pessoa, podendo ser aguda ou crônica. ${ }^{12}$ Trata-se de uma manifestação subjetiva, que envolve mecanismos físicos, psíquicos e culturais. A dor em idosos é um sério problema de saúde pública, que necessita ser diagnosticado, mensurado, avaliado e devidamente tratado pelos profissionais de saúde, uma vez que são esses os agentes capazes de, através de intervenções, minimizar a morbidade e melhorar a qualidade de vida desses indivíduos. ${ }^{10}$

A dor confronta o idoso em sua fragilidade e ameaça sua segurança, autonomia e independência, impedindo muitas vezes sua capacidade de realizar as atividades da vida diária, bem como limitando sua capacidade de interação e convívio social - situações que diminuem consideravelmente sua qualidade de vida. A dor aguda surge de forma súbita e tem como função alertar o indivíduo para o perigo de uma lesão. A dor crônica é considerada aquela com duração maior de seis meses, ou que ultrapassa o período usual de recuperação esperado para a causa desencadeante da dor. ${ }^{13}$

Fatores como depressão, incapacidade física e funcional, dependência, afastamento social, mudanças na sexualidade, alterações na dinâmica familiar, desequilíbrio econômico, desesperança, sentimento de morte e outros, encontram-se associados a quadros de dor crônica. A dor passa a ser o centro, direciona e limita as decisões e comportamentos do indivíduo.
Acarreta, ainda, fadiga, anorexia, alterações do sono, constipação, náuseas, dificuldade de concentração, entre outros. A impossibilidade de controlá-la traz sempre sofrimento físico e psíquico. Todos esses fatores associados parecem aumentar a morbidade entre os idosos e onerar o sistema de saúde. As consequências biopsicossociais da dor crônica enfatizam a importância do dimensionamento da sua prevalência visando ao planejamento de medidas para seu controle e tratamento. ${ }^{11}$

\section{Dor lombar e envelhecimento}

O número de idosos no Brasil, que era 3 milhões em 1960, passou para 7 milhões em 1975 e 20 milhões em 2008. Estima-se que em 2025, $15 \%$ da população brasileira estará com mais de 60 anos, ocorrendo um aumento médio de $6,5 \%$ de idosos ao ano e, ao mesmo tempo, uma redução nos números absolutos de jovens entre 0 e 14 anos. ${ }^{14-16}$ Essas cifras levarão o país a ocupar a sexta posição entre os países com maior número de idosos do mundo. ${ }^{11}$

Os sinais do envelhecimento se traduzem com o decorrer da idade e acarretam a redução da elasticidade dos tecidos moles; diminuição da estatura dos discos intervertebrais; menor capacidade articular de absorver pressões, tornando-se mais rígidas; cartilagens menos elásticas; menor poder muscular, levando à desaceleração dos movimentos e à perda da coordenação, dentre outros acometimentos. ${ }^{17}$

A coluna do homem é constituída de vértebras, ligamentos, músculos e discos intervertebrais. Intercalados entre os corpos vertebrais estão os discos, os quais se encontram relacionados com as funções de amortecimento de pressões e sustentação de peso. ${ }^{17} \mathrm{~A}$ eficiência biomecânica do disco intervertebral diminui com o envelhecimento, devido à sua desidratação e ao aumento do estresse mecânico, comprometendo a integridade desse verdadeiro amortecedor de choques e presdipondo-o à herniação. Concomitantemente, as alterações ósseas decorrentes do processo de envelhecimento, juntamente com desordens estruturais e desvios mecânicos, tornam as patologias degenerativas o principal fator etiológico das lombalgias na fase senil, sendo a osteoartrose o maior motivo de consultas médicas. ${ }^{6}$

A degeneração da coluna vertebral inerente ao processo de envelhecimento, juntamente com o maior tempo de exposição a sobrecargas ao longo da vida, pode predispor o idoso a ser acometido pela dor lombar. ${ }^{9}$ Devido à complexidade das lombalgias, é possível classificá-las etiologicamente como estruturais, traumáticas, musculoesqueléticas, degenerativas, reumáticas, provocadas por defeitos congênitos, 
inflamatórias, neoplásicas, viscerais reflexas, decorrentes de doenças ósseas; e metabólicas. ${ }^{18,19}$

A dor lombar crônica é uma das disfunções musculoesqueléticas mais comuns no mundo, acometendo cerca de $70 \%$ a $85 \%$ da população em alguma época da vida, sendo hoje, uma das principais queixas na clínica diária, podendo resultar em consequências que incluem absenteísmo no trabalho, fatores psicológicos, sociais e econômicos que interferem nas atividades básicas de vida diária, além de relação com condições cardiometabólicas. ${ }^{20,21}$

As lombalgias relacionadas ao compartimento anterior da coluna podem ser originadas do corpo vertebral, como nas infecções, neoplasias primitivas ou metastáticas, fraturas de compressão etc., ou do disco intervertebral, como nas discopatias degenerativas, nas infecciosas ou nas hérnias discais. A estenose do canal vertebral e a síndrome de compressão radicular em nível dos forames de conjugação são as causas mais frequentes de algias vertebrais do compartimento médio, enquanto o compartimento posterior está relacionado às lombalgias originadas das partes moles, como entorses, distensões ou estiramentos musculo ligamentares, a par das artropatias interapofisárias associadas à osteoartrose, artrite reumatoide e espondilite anquilosante. ${ }^{17} \mathrm{O}$ tratamento pode ser realizado de modo que evite a realização de cirurgias no idoso. ${ }^{22}$

Em um estudo de Kleinpaul et al. (2008), ${ }^{9}$ relacionando dor na lombar e na extremidade inferior do corpo em indivíduos de ambos os sexos, subdivididos pela faixa etária em adultos de meia-idade e idosos, foi encontrado que mulheres de meia-idade e idosas têm uma capacidade funcional diminuída em relação aos homens e um alto índice na escala visual analógica (EVA), esta escala consiste em auxiliar na aferição da intensidade da dor no indivíduo, estatisticamente significativo quando comparado a homens. Dentro deste contexto, alguns autores supõem que as mulheres estão expostas a riscos maiores que os homens devido a particularidades anatomofuncionais que, quando somadas, podem corroborar o surgimento de lombalgias. Dentre tais aspectos, podem ser destacados os seguintes: menor estatura, menor massa muscular, menor densidade óssea, fragilidade articular e menor adaptação ao esforço físico. Além disso, as cargas ergonômicas impostas pela realização das tarefas domésticas, além do trabalho fora de casa, potencializam este risco. ${ }^{6}$ Também são considerados fatores de risco associados à dor lombar, o IMC aumentado e as expectativas negativas quanto à reabilitação. ${ }^{23}$

A crescente incidência da dor lombar e suas implicações socioeconômicas levaram a busca de melhores métodos de diagnóstico, tratamento e especialmente, de avaliação da incapacidade. A avaliação com testes de flexão-extensão é apontada na literatura como eficaz no diagnóstico e para determinar os limitantes da dor lombar crônica no idoso. ${ }^{24,25}$ Nos últimos anos, observa-se uma forte tendência à utilização de medicina complementar alternativa, com o intuito de dar ênfase a tratamentos manuais incluindo ajustamentos articulares e manipulação de tecidos moles. ${ }^{26}$ Estudos demonstram que pacientes mais velhos que recebem tratamento quiroprático, por exemplo, tendem a utilizar menos medicações prescritas, estão menos propensos a usar recursos de enfermagem, e relatam, com maior frequência, um bom estado de saúde. ${ }^{27}$

\section{Fatores sociais e incapacidades decorrentes da lombalgia}

No Brasil, segundo dados da Pesquisa Nacional por Amostra de Domicílios (Pnad), em 2007 já havia 20 milhões de idosos, correspondendo a 10,5\% da população. Nesse contexto, as enfermidades crônicas associadas ao envelhecimento surgem como preocupação, sobretudo, relacionadas à qualidade de vida neste período. Isso porque tais enfermidades apresentam relação estatisticamente significante com o nível de dependência funcional dos idosos. Essas enfermidades podem estar relacionadas a relatos de dor, os quais, por sua vez, podem relacionar-se à incapacidade funcional. A relação entre dor e dependência funcional evidencia-se pelo comportamento da dor durante a execução de atividades da vida diária por idosos. ${ }^{28}$

Nem todos vivem o processo de envelhecimento da mesma maneira, uma vez que esse fenômeno está estreitamente relacionado às formas materiais e simbólicas que identificam socialmente cada indivíduo. ${ }^{4}$ Sabe-se hoje que a dor, em especial a dor crônica, é crescente, talvez devido aos novos hábitos de vida, à maior longevidade do indivíduo e provavelmente ao reconhecimento de novas condições álgicas e de aplicações de novos conceitos que traduzem seu significado. ${ }^{20}$ Vlaeyen e Crombez (1999) ${ }^{29}$ salientaram que a dor vai além de uma experiência emocional podendo ser considerada como uma experiência multidimensional e, que a antecipação da dor e o medo devem ser relacionados com a persistência da incapacidade física em estágio crônico na dor lombar.

A dor lombar crônica resulta em diminuição do constructo "capacidade funcional" no trabalho e na realização das atividades de vida diárias, afetando assim a qualidade de vida desses indivíduos. Diante disso, a necessidade de mudanças na conduta relacionada à incapacidade tem sido objeto de preocupação pelo 
alto custo com os cuidados em saúde, as implicações socioeconômicas e a recorrência elevada. ${ }^{20}$

Abreu (2006), ${ }^{30}$ em seu estudo, encontrou uma forte correlação entre intensidade da dor com grau de incapacidade, sugerindo que a intensidade da dor pode ser um fator importante na manutenção e aumento da incapacidade. ${ }^{30}$ Alguns autores relataram que não só a intensidade da dor está relacionada à incapacidade, mas também, a duração da dor pode contribuir para depressão e crenças de medo, favorecendo o desenvolvimento de uma condição de incapacidade prolongada com restrição nas atividades de vida diárias e no trabalho. ${ }^{31-34}$

Kovacs et al. (2005), ${ }^{35}$ em outra pesquisa objetivando explorar e verificar diferenças entre os estágios agudos e subagudos baseados na correlação entre dor, incapacidade e qualidade de vida em pacientes no início do estágio crônico, observaram que a influência da dor e incapacidade na qualidade de vida continua aumentando até ou mais sessenta dias, entretanto, a influência da dor na incapacidade aumenta rapidamente nos primeiros quatorze dias. Esses autores salientaram ainda que nesses pacientes a dor não desaparece e a tendência natural é que com o tempo os mesmos acostumem com a dor, tornando-a um efeito relativo na incapacidade e qualidade de vida.

De acordo com Kovacs et al. (2004), ${ }^{36}$ não só a dor e a incapacidade determinam a qualidade de vida em pacientes com dor lombar crônica, mas outros fatores não relacionados a essa condição de saúde podem influenciar a qualidade de vida, como fatores pessoais, familiares e econômicos, ansiedade, depressão e outras situações da vida. $O$ estudo de revisão $0^{37}$ aponta o excesso de peso como um fator de grande influência na dor lobar ao caminhar e subir escadas. Portanto, tanto fatores biomecânicos podem influenciar a dor enquanto fatores psicossociais podem influenciar o desenvolvimento e duração da incapacidade. Nesse cenário de condição de saúde pública, com grande prejuízo na capacidade funcional, diminuição na qualidade de vida e aumento de consequências socioeconômicas, estratégias de prevenção e/ou tratamento vem sendo utilizadas, mas ainda representam um grande desafio para toda sociedade. ${ }^{20,37}$

\section{CONCLUSÕES}

A lombalgia pode levar à incapacidade funcional, restringir a qualidade de vida do idoso na comunidade e ameaçar sua independência. A alta prevalência encontrada em grande parte dos estudos atuais é um fator preocupante e que deve auxiliar no desenvolvimento de estratégias direcionadas com o intuito de proporcionar intervenção, tanto para tratamento quanto para a prevenção de novos acometimentos, a fim de garantir o bem-estar dessa população no Brasil e no mundo.

Apesar da magnitude do impacto social e econômico, apontada em estudos internacionais, no Brasil há escassez de estudos sobre prevalência de lombalgia em idosos. Esse fato dificulta a sensibilização de profissionais da área de saúde, a alocação de recursos humanos e materiais, e a criação de estratégias visando ao controle da dor lombar nessa população, tornando emergente a necessidade de estudos epidemiológicos com este enfoque.

\section{REFERÊNCIAS}

1. Rossi E, Sader CS. Envelhecimento do Sistema Osteoarticular. In: Freitas EV et al. Tratado de Geriatria e Gerontologia. Rio de Janeiro: Guanabara Koogan; 2006. p. 792-7.

2. Lucena LSC. Hidroterapia na qualidade de vida na lombalgia senil [Trabalho de conclusão de curso]. Goiania (GO): Universidade Paulista; 2011.

3. Mazo G, Lopes M, Benedetti T. Atividade física e o idoso: concepção gerontológica. 2ª ed. Porto Alegre: Sulina; 2004.

4. Grassi C. Dor lombar em idosos praticantes de exercício físico [Trabalho de conclusão de curso]. Ijuí (RS): Universidade Regional do Noroeste do Estado do Rio Grande do Sul; 2012

5. Leopoldino AAO, Diz JMB, Martins VT et.al. Prevalence of low back pain in older Brazilians: a systematic review with meta-analysis. Rev. Bras. Reumatol. 2016;56(3):258-269.

6. Reis LA, Mascarenhas CHM, Filho LENM et al. Lombalgia na terceira idade distribuição e prevalência na Clínica Escola de Fisioterapia da Universidade Estadual do Sudoeste da Bahia. Rev Bras Geriatr Gerontol. 2008;11(1):93-103.

7. Castro MG. A coluna lombar do idoso. Rev Bras Ortop. 2000;35(11/12):423-5.

8. Brazil AV, Ximenes AC, Radu AS, et al. Diagnóstico e tratamento das lombalgias e lombociatalgias. Rev Bras Reumat. 2004;44(6):419-25.

9. Kleinpaul JF, Mann L, Teixeira CS et al. Dor Lombar e exercício físico. Uma revisão. Efdeportes - Rev Dig B Aires [periódico online]. 2008 [capturado 2016 Ago 16]; 127: Disponível em http://www.efdeportes.com/efd127/dorlombar-e-exercicio-fisico.htm

10. Celich KLSC, Galon C. Dor crônica em idosos e sua influência nas atividades da vida diária e convivência social. Rev Bras Geriatr Gerontol. 2009;12(3):345-59.

11. Dellaroza MSG, Pimenta CAM, Matsuo T. Prevalência e caracterização da dor crônica em idosos não institucionalizados. Cad Saude Publica. 2007;23(5):1151-61.

12. Silvestre JA, Kalache A, Ramos LR et al. O envelhecimento populacional brasileiro e o setor saúde. Arq Geriatr Gerontol. 1996;1:81-9.

13. Silva MCS. Dor no idoso. In: Terra NL. Envelhecimento bem sucedido. Porto Alegre: EDIPUCRS; 2002. p. 161-5.

14. Baptista RR, Vaz MA. Arquitetura muscular e envelhecimento: adaptação funcional e aspectos clínicos; revisão da literatura. Fisiot Pesq. 2009;16(4):368-73. 
15. Matsudo SM, Neto VKR, Barros TL. Impacto do envelhecimento nas variáveis antropométricas, neuromotoras e metabólicas da aptidão física. Rev Bras Cien Mov. 2000;8(4):21-32.

16. Rebelatto JR, Calvo JI, Orejuela JR et al. Influência de um programa de atividade física de longa duração sobre a força muscular manual e a flexibilidade corporal de mulheres idosas. Rev Bras Fisioter. 2006;10(1):127-32.

17. Silveira MM, Pascualotti A, Colussi EL et al. Abordagem fisioterápica da dor lombar crônica no idoso. Rev Bras Cienc Saúde. 2010;25.

18. Costa D, Palma A. O efeito do treinamento contra resistência na síndrome da dor lombar. Rev Portug Ciencias Desporto. 2005;5(2):224-34.

19. Ferreira Caetano L. Aquatic Therapy in low back pain reduction evaluated through hydroxiproline levels. Fitness Perform J. 2006;5(1):39-43.

20. Bento AAC, Paiva ACS, Siqueira FB. Correlação entre incapacidade, dor - Roland Morris, e capacidade funcional SF-36 em indivíduos com dor lombar crônica não específica. Rev E-scientia. 2009;2(1).

21. Scherer M, Hansen H, Gensichen J et.al; Association between multimorbidity patterns and chronic pain in elderly primary care patients: a cross-sectional observational study. BMC Family Practice. 2016;17:68.

22. Fritz JM, Rundell SD, Dougherty P et.al. Deconstructing Chronic Low Back Pain in the Older Adult-Step by Step Evidence and ExpertBased Recommendations for Evaluation and Treatment. Part VI: Lumbar Spinal Stenosis. Pain Medicine. 2016;17:501-10.

23. Enthoven WTM, Koes BW, Bierma-Zeinstra SMA et al. Defining trajectories in older adults with back pain presenting in general practice. Age and Ageing. 2016; $0: 1-5$.

24. Kienbacher T, Fehrmann E, Habenicht R et al. Diagnostic value of trunk flexion-extension testing in old chronic low back pain patients. Eur Spine J. 2016;1-8.

25. Kienbacher T, Fehrmann E, Habenicht R et.al. Age and gender related neuromuscular pattern during trunk flexion-extension in chronic low back pain patients. Journal of NeuroEngineering and Rehabilitation. 2016; 13:6.
26. World Federation of Chiropractic. Definition of chiropractic. World Federation of Chiropractic [cited 2016 Aug 16]; Available from: http:/www.wfc.org/website/WFC/website. nsf/WebPage/DefinitionOfChiropractic?OpenDocument

27. Coulter I, Hurwitz E, Aronow H et al. Chiropratic patients in a comprehensive home-based geriatric assessment, followup and health promotion program. Topics Clin Chiropractic. 1996;3(2):46-55.

28. Maraschin R, Vieira PS, Leguisamo CP et al. Dor lombar crônica e dor nos membros inferiores em idosas etiologia em revisão. Fisioter Mov. 2010;23(4):627-39.

29. Vlaeyen JWS, Crombez G. Fear of movement/(re)injury, avoidance and pain disability in chronic low back pain patients. Manual Therapy. 1999;4(4):187-95.

30. Abreu AM. Tradução e adaptação cultural para a língua portuguesa do Fear Avoidance Beliefs Questionnaire (FABQ) em portadores de dor lombar crônica [dissertação]. Franca (SP): Universidade de Franca; 2006.

31. Jacob T, Baras M, Zeev A et al. Low back pain: reliability of a set of pain measurement tools. Arch Phys Med Rehabilitation. 2001;82(6):735-42.

32. Salvetti MG, Pimenta CAM. Dor crônica e a crença de auto-eficácia. Rev Esc Enferm USP. 2007;41(1):135-40.

33. Verbunt JA, Westerterp KR, van der Heijden GJ et al. Physical activity in daily life in patients with chronic low back pain. Arch Phys Med Rehabilitation. 2001;82(6): 726-30.

34. Woby SR, Watson PJ, Roach NK et al. Are changes in fearavoidance beliefs, catastrophizing, and appraisals of control, predictive of changes in chronic low back pain and disability? Eur J Pain. 2004;8(3):201-10.

35. Kovacs FM, Abraira V, Zamora J, Fernández C. The transition from acute to subacute and chronic low back pain - a study based on determinants of quality of life and prediction of chronic disability. Spine. 2005;30:1786-92.

36. Kovacs FM, Abraira V, Zamora J et al. Correlation Between pain, disability and quality of life in patients with common low back pain. Spine. 2004;29:206-10.

37. Vicent HK, Seay NA, Montero C et al. Functional Pain Severity and Mobility in Overweight Older Men and Women with Chronic Low Back Pain: Part I. Am J Phys Med Rehabil. 2013;92(5):430-8. 